COMMUNICATIONS IN

NUMBER THEORY AND PHYSICS

Volume 7, Number 3, 397-410, 2013

\title{
Functional equations and ladders for polylogarithms
}

\author{
HERBERT GANGL
}

\begin{abstract}
We give a number of $\mathcal{S}_{3}$-symmetric functional equations for polylogarithms up to weight 7 . This allows one to obtain the first proven ladder relations, à la Lewin, of weight 6 and 7 .
\end{abstract}

\section{Motivation}

Polylogarithms appear in many contexts within mathematical physics, like in dimensional regularization expansions or when determining analytic solutions of various Feynman integrals in quantum field theory; e.g., the dilogarithm appeared already in the famous paper by t'Hooft and Veltman [21], and Ussyukina and Davydychev [29], Equation (30), encountered all $m$ logarithms $(n \leq m \leq 2 n)$ in a closed expression for the " $n$-box" diagram (for a more recent update cf. [28]), as well as in conformal field theory (the dilogarithm plays a crucial role in a conjecture of Nahm [27] characterizing rational CFTs) or when considering expansions of hypergeometric functions (cf. e.g., [22]). Even more closely related to our results below, (multiple) polylogarithms and their special values have occurred, among many others, in various ways in work of Broadhurst and Kreimer (e.g., [6]), occasionally even in connection with ladder relations (cf. [4,5]) as defined below. Recently, when calculating the two-loop hexagon Wilson loop in $\mathcal{N}=4$ supersymmetric Yang-Mills theory, Del Duca, Duhr and Smirnov [10] were led to a long expression in polylogarithms that has been subsequently enormously simplified by Goncharov et al. [20] using Goncharov's notion of a symbol attached to a (multiple) polylogarithm (the first combinatorial description being given, under the name $\bigotimes^{m}$-invariant, in [19], Section 4.4). Subsequent papers, especially in particle physics, by many more authors (e.g., $[7,8,11-13,17])$ have studied similar expressions in various contexts. While the symbols somehow aim to circumvent having to apply functional equations, it seems still conceivable that in these contexts insight into functional 
equations for the polylogarithms involved might be useful to reduce the ensuing - typically very complicated — expressions considerably.

Functional equations of polylogarithms play also a pivotal role in a more abstract context when trying to define an explicit version of the (odd indexed) algebraic $K$-groups $K_{2 m-1}(F)$ of a number field $F$. The latter can conjecturally be written as a subquotient of the free abelian group on $F$ (pioneered by Bloch [3] in the dilogarithm case and generalized by Zagier [31] and by Goncharov [18] for higher $m$ ), and the group of relations in that description is expected to encode all the functional equations of the $m$-logarithm.

In 1840, Kummer [23] gave non-trivial functional equations for polylogarithms $L i_{m}(z)=\sum_{n \geq 1} z^{n} / n^{m}$ up to weight $m=5$, where results had previously been known only up to $m=3$. He mentioned "peculiar difficulties" ("eigenthümliche Schwierigkeiten") that arise when trying to extend the results to $m>5$. In fact, Wechsung proved [30] that the type of functional equation that Kummer had found does not extend to $m>5$.

In the eighties, Lewin and his coauthors $([1,26])$ tried several approaches to conquer what he called the "trans-Kummer region" $m>5$ (cf. e.g., [1], p. 11), and they indeed found new functional equations, but all results were ultimately confined to the same range $m \leq 5$. On the way, Lewin discovered interesting special relations of the form $\sum_{j} n_{j} L i_{m}\left(\alpha^{j}\right)=0\left(n_{j} \in \mathbf{Q}\right)$, for certain algebraic numbers $\alpha$. He realized that such relations, which he dubbed "ladders", were consequences of a certain intrinsic property of such an $\alpha$, viz. the property that it satisfies many different "cyclotomic relations" (loc.cit.), which are equations of the form $\prod_{r}\left(1-\alpha^{r}\right)^{\nu_{r}}= \pm \alpha^{N}$ where $r, \nu_{r}$ and $N$ are integers. This insight enabled him to conjecture certain ladders even up to weight $m=9$ (he used the terminology order in place of the now more common notion of weight). By cleverly specializing and combining old and new functional equations, he was able to prove quite a number of his conjectured ladders, but was again confined to weights $\leq 5$.

The first functional equations for $m=6$ and $m=7$ were constructed in [14-16], and no examples of higher weight are known. In this paper, we describe a collection of functional equations for polylogarithms up to this weight that have a very specific symmetry: the arguments (in one variable $t$ ) involve only the three factors $t, 1-t$ and $1-t(1-t$ ) (with roots 0,1 and the primitive sixth roots of unity, respectively), and each given equation is invariant under the action of the symmetric group $\mathcal{S}_{3}$.

As a by-product, the equations for weight 6 and 7 allow, after specialization, to prove the first ladders in that range. 


\section{Zagier's criterion for functional equations of polylogarithms}

In his seminal papers [31,32], Zagier described a criterion for functional equations for polylogarithms. More precisely, he first gave a single-valued function $\mathcal{L}_{m}(z)=\Re_{m}\left(\sum_{k=0}^{m-1} \frac{2^{k} B_{k}}{k !} \log ^{k}|z| L i_{m-k}(z)\right)$ (denoted by $P_{m}(z)$ in [31]) attached to the (multivalued) function $L i_{m}(z)$, where $\Re_{m}$ denotes the real part for $m$ odd and the imaginary part for $m$ even, and the $B_{k}$ denote the Bernoulli numbers. This function now satisfies "clean" functional equations, i.e., without invoking products of lower weight polylogarithms as occur typically — and in abundance - for $L i_{m}$-equations (cf. e.g., almost any functional equation in [24]). Furthermore, one can give a very useful characterization for them which we describe in the following subsection.

\subsection{Higher Bloch conditions}

For a field $F$, let $\beta_{m}^{F}$ be the map

$$
\beta_{m}^{F}: \quad \mathbf{Z}[F] \longrightarrow \bigotimes^{m-2} F^{\times} \otimes \bigwedge^{2} F^{\times}
$$

defined as $\beta_{m}^{F}([0])=\beta_{m}^{F}([1])=0$, and on generators $[x](x \neq 0,1)$ as follows:

$$
\beta_{m}^{F}([x])=x \otimes \cdots \otimes x \otimes(x \wedge(1-x)) .
$$

For $m=2$, this map was related to the dilogarithm in Bloch's seminal paper [3].

We say that a combination $\xi \in \mathbf{Z}[F]$ satisfies the $m$-th higher Bloch condition simply if it lies in $\operatorname{ker} \beta_{m}^{F}$. This fits very well with the above one-valued function $\mathcal{L}_{m}(z)$ :

Theorem 1 (Zagier, [31]). Let $F$ be a subfield of $\mathbf{C}(t)$ then for $\xi \in \mathbf{Z}[F]$ we have

$$
\xi \in \operatorname{ker} \beta_{m}^{F} \Longrightarrow \mathcal{L}_{m}(\sigma(\xi))=\text { constant }
$$

for any embedding $\sigma: F \hookrightarrow \mathbf{C}(t)$.

Here we extend the definition of $\mathcal{L}_{m}$ as well as of $\sigma$ to all of $\mathbf{Z}[F]$ by linearity, i.e.,

$$
\mathcal{L}_{m} \circ \sigma\left(\sum_{i} n_{i}\left[x_{i}\right]\right)=\sum_{i} n_{i} \mathcal{L}_{m}\left(\sigma\left(x_{i}\right)\right)
$$


In this way, the problem of finding functional equations with given arguments $x_{i}$ is reduced to a problem in linear algebra and the hard part is to find a suitable list of potentially good arguments.

\subsection{A rich collection of arguments}

A particularly good collection of arguments for functional equations (in one variable $t$ ) turns out to be given by

$$
\left\{ \pm t^{a_{1}}(1-t)^{a_{2}}(1-t(1-t))^{a_{3}} \mid a_{i} \in \mathbf{Z}\right\} .
$$

It is convenient to introduce new variables

$$
u_{1}(t)=\frac{-t}{1-t(1-t))}, \quad u_{2}(t)=\frac{-(1-t)}{1-t(1-t)}, \quad u_{3}(t)=\frac{t(1-t)}{1-t(1-t)}
$$

and then to rewrite the above expressions as

$$
\left\{ \pm u_{1}(t)^{\alpha_{1}} u_{2}(t)^{\alpha_{2}} u_{3}(t)^{\alpha_{3}} \mid \alpha_{i} \in \mathbf{Z}\right\}
$$

for suitable $\alpha_{i}$, since then a further $\mathcal{S}_{3}$-symmetry becomes apparent. The two involutory automorphisms induced by $t \mapsto \frac{1}{t}$ and $t \mapsto 1-t$, respectively, generate this $\mathcal{S}_{3}$-action on the set of those arguments by simply permuting the exponents. Any of the arguments can hence be encoded by a triple of exponents, together with a sign. There are many functional equations for $m \leq 7$, in the exponent range $\left|\alpha_{i}\right| \leq 6$, which carry the above symmetry. All the ones that were found have arguments chosen from the following list $\mathcal{A}$ which represents $32 \mathcal{S}_{3}$-orbits in $\mathbf{Z}[\mathbf{Q}(t)]$ :

$$
\begin{aligned}
\mathcal{A}=\{ & (-, 2,-2,3),(+, 0,5,0),(-, 6,-1,-1),(+, 3,0,0), \\
& (+, 0,-3,3),(-,-3,6,-3),(-,-3,3,3),(+, 0,-5,5), \\
& (+, 4,-1,0),(+,-3,4,4),(+, 3,0,-2),(-,-1,2,-1), \\
& (+, 0,1,1),(-, 2,0,-2),(+, 1,0,-1),(-, 1,0,-1), \\
& (+,-2,-2,3),(-,-1,3,-1),(+,-4,-1,4),(-,-2,-2,5), \\
& (-, 2,-1,1),(-,-2,-1,3),(-, 2,0,-1),(+, 2,0,-1), \\
& (-,-2,2,2),(+, 2,-1,-1),(-, 2,-1,-2),(-, 0,1,0), \\
& (+, 0,1,0),(-,-1,1,1),(+, 1,-1,-1),(-, 1,1,0)\} .
\end{aligned}
$$

The factors of $1-x$ where $x$ runs through those arguments can be found in the $\mathcal{S}_{3}$-orbits of the following list (where $T=1-t(1-t)$ ):

$$
\left\{t, T, 1+t, 1+t(1-t), 1+\frac{1}{T}, 1+\frac{t}{T}, 1+\frac{1-t}{t T}, 1+\frac{t(1-t)^{2}}{T^{2}}, 1-\frac{t^{2}(1-t)}{T^{2}}\right\} .
$$




\subsection{The functional equations}

Due to the symmetry just explained we focus on $\mathcal{S}_{3}$-invariant functional equations and introduce the shorthand

$$
\left[\left( \pm, \alpha_{1}, \alpha_{2}, \alpha_{3}\right)\right]:=\sum_{\sigma \in \mathcal{S}_{3}}\left[ \pm \prod_{i=1}^{3} u_{i}(t)^{\alpha_{\sigma(i)}}\right]
$$

Using this notation, the functional equations can be given in concise form, with coefficients taken from the tables below. We first state the results for combinations satisfying the higher Bloch conditions.

Theorem 2. For $m \in \mathbf{N}$, let $\kappa_{m}=\operatorname{ker}\left(\beta_{m}^{\mathbf{Q}(t)}\right)^{\mathcal{S}_{3}}$ be the space of $\mathcal{S}_{3}-$ invariant elements in the kernel of the map $\beta_{m}^{\mathbf{Q}(t)}$. Then we have the following bounds on the ranks of $\kappa_{m}$ for $m=4,5,6,7$.

\begin{tabular}{|c||cccc|}
\hline$m$ & 4 & 5 & 6 & 7 \\
\hline rank $\kappa_{m}$ & $\geq 11$ & $\geq 9$ & $\geq 4$ & $\geq 2$ \\
\hline
\end{tabular}

The corresponding elements are given by

$$
\sum_{a \in \mathcal{A}} c_{j}^{(m)}(a)[a]
$$

with the coefficients $c_{j}^{(m)}=\left\{c_{j}^{(m)}(a)\right\}_{a \in \mathcal{A}}$ as in tables 1-3 below.

The proof that the given elements are indeed in the kernel of $\beta_{m}^{F}$ is a tedious and mechanical task, which is best left to a computer. One determines all the factors occurring in a factorization of $x$ and $1-x$, where $x$ runs through all the corresponding arguments in an equation and then checks that all the terms in the ensuing image under $\beta_{m}^{F}$ do cancel. Using the $\mathcal{S}_{3^{-}}$ symmetry involved, one can cut down on the actual calculations, but they are still too cumbersome to give in detail.

Corollary 3. There are at least 2 (resp., 4, 9, 11) linearly independent $\mathcal{S}_{3}$ symmetric functional equations for $\mathcal{L}_{7}$ (resp., $\mathcal{L}_{6}, \mathcal{L}_{5}, \mathcal{L}_{4}$ ) with arguments encoded (up to permutation) by $\mathcal{A}$.

We remark that the two functional equations for $\mathcal{L}_{7}$ do not seem to follow individually from the 2 -variable equation for $\mathcal{L}_{7}$ given in [16], but the linear combination of the two which cancels the constant terms is a specialization of that equation. 
Example. We spell out some equations corresponding to the columns of table 1 . The last one, $c_{11}^{(4)}$, gives

$$
2[(+, 2,-1,-1)]+6[(-, 0,1,0)]+3[(+, 1,-1,-1)] \in \operatorname{ker} \beta_{4}^{F}
$$

for $F=\mathbf{Q}(t)$. This is equivalent to the 9-term equation for $\mathcal{L}_{4}$ cited in [32], Section 7 . The second-to-last column gives another element in that kernel,

$$
\begin{aligned}
{[(-,-2,2,2)] } & +4[(-, 2,-1,-2)]-6[(-, 0,1,0)] \\
& -12[(+, 0,1,0)]-2[(+, 1,-1,-1)]
\end{aligned}
$$

Explicitly, but with less apparent symmetry, this can be written as

$$
\begin{aligned}
& 2\left(\mathcal{L}_{4}(\right.\left.\left.-\frac{t^{4}}{T^{2}}\right)+\mathcal{L}_{4}\left(-\frac{(1-t)^{4}}{T^{2}}\right)+\mathcal{L}_{4}\left(-\frac{1}{T^{2}}\right)\right) \\
&+ 4\left(\mathcal{L}_{4}\left(-\frac{(1-t) T}{t^{3}}\right)+\mathcal{L}_{4}\left(-\frac{t T}{(1-t)^{3}}\right)+\mathcal{L}_{4}\left(\frac{T}{t^{3}}\right)\right. \\
&\left.+\mathcal{L}_{4}\left(\frac{T}{(1-t)^{3}}\right)+\mathcal{L}_{4}((1-t) T)+\mathcal{L}_{4}(t T)\right) \\
&- 12\left(\mathcal{L}_{4}\left(-\frac{t(1-t)}{T}\right)+\mathcal{L}_{4}\left(\frac{t}{T}\right)+\mathcal{L}_{4}\left(\frac{1-t}{T}\right)\right) \\
&- 24\left(\mathcal{L}_{4}\left(\frac{t(1-t)}{T}\right)+\mathcal{L}_{4}\left(-\frac{t}{T}\right)+\mathcal{L}_{4}\left(-\frac{1-t}{T}\right)\right) \\
&-4\left(\mathcal{L}_{4}\left(\frac{T}{t^{2}}\right)+\mathcal{L}_{4}\left(\frac{T}{(1-t)^{2}}\right)+\mathcal{L}_{4}(T)\right)=0
\end{aligned}
$$

where $T=1-t(1-t)$ as before.

Note that there are shorter combinations like $\frac{1}{10}\left(c_{5}^{(5)}-3 c_{9}^{(5)}\right)$ which is essentially (7.99) in [24].

The constant of Theorem 1 is zero for each $c_{j}^{(m)}$ for even $m$, while for $m=$ 5 or 7 the constants can be obtained by specialising $t$ to 1 , say, and turn out to be of the form $\lambda\left(c_{j}^{(m)}\right) \zeta(m)$ with $\lambda\left(c_{j}^{(m)}\right) \in \mathbf{Q}$ and $\zeta(m)=\mathcal{L}_{m}(1)$ denoting the corresponding Riemann zeta value. The corresponding values of $\lambda(=$ $0,0,0,0,1662,378,4230,-126$ and 414 for $m=5$ and $-\frac{25461}{4}$ and $-\frac{54495}{4}$ for $m=7$, respectively) are given in the last lines of tables 2 and 3, respectively. Note that certain $a \in \mathcal{A}$ occur with non-trivial coefficient only for odd $m$ as the inversion relation annihilates the sum over the corresponding orbit for even $m$. Moreover, in order to display how the order of the columns has been chosen we indicate the first non-zero value in each column of tables 1 and 2 in bold face. 


\subsection{The tables}

\subsubsection{Functional equations for $m=4$}

Table 1: Generators for $\operatorname{ker}\left(\beta_{4}^{\mathbf{Q}(t)}\right)^{\mathcal{S}_{3}}$ in Theorem 2

\begin{tabular}{|c|c|c|c|c|c|c|c|c|c|c|c|c|c|}
\hline & $a$ & & $c_{1}^{(4)}$ & $c_{2}^{(4)}$ & $c_{3}^{(4)}$ & $c_{4}^{(4)}$ & $c_{5}^{(4)}$ & $c_{6}^{(4)}$ & $c_{7}^{(4)}$ & $c_{8}^{(4)}$ & $c_{9}^{(4)}$ & $c_{10}^{(4)}$ & $c_{11}^{(4)}$ \\
\hline$(-, \quad 2$, & -2 & 3) & 2 & 0 & 0 & 0 & 0 & 0 & 0 & 0 & 0 & 0 & 0 \\
\hline,$+ \quad 0$, & 5 & 0) & 0 & 1 & 0 & 0 & 0 & 0 & 0 & 0 & 0 & 0 & 0 \\
\hline,$- \quad 6$ & -1 & $-1)$ & 0 & 0 & 1 & 0 & 0 & 0 & 0 & 0 & 0 & 0 & 0 \\
\hline,$- \quad 3$ & 0 & 0) & 0 & 0 & 0 & 3 & 0 & 0 & 0 & 0 & 0 & 0 & 0 \\
\hline,$+ \quad 0$, & -3 & & 0 & 0 & 0 & 0 & 0 & 0 & 0 & 0 & 0 & 0 & 0 \\
\hline,--3 , & 6 & $-3)$ & 0 & 0 & 0 & 0 & 3 & 0 & 0 & 0 & 0 & 0 & 0 \\
\hline$(-,-3$, & 3 & 3) & 0 & 0 & 0 & 0 & 0 & 3 & 0 & 0 & 0 & 0 & 0 \\
\hline$-\quad 0$ & -5 & & 0 & 0 & 0 & 0 & 0 & 0 & 0 & 0 & 0 & 0 & 0 \\
\hline$-\quad 4$, & -1 & 0) & 0 & 0 & 0 & 0 & 0 & 0 & 2 & 0 & 0 & 0 & 0 \\
\hline,+-3 , & 4 & 4) & 0 & 0 & 1 & 1 & -1 & -1 & 0 & 0 & 0 & 0 & 0 \\
\hline,$+ \quad 3$, & 0 , & $-2)$ & 0 & -10 & 0 & 0 & 0 & 0 & -6 & 0 & 0 & 0 & 0 \\
\hline,--1, & 2 & $-1)$ & 0 & 0 & 0 & 0 & -81 & 0 & 0 & 0 & 0 & 0 & 0 \\
\hline,$+ \quad 0$, & 1 & 1) & 0 & -30 & 0 & 0 & 0 & 0 & -6 & 0 & 0 & 0 & 0 \\
\hline,$- \quad 2$, & 0 , & $-2)$ & 0 & 0 & 0 & 0 & 0 & 0 & 0 & 0 & 0 & 0 & 0 \\
\hline,$- \quad 1$, & 0 & $-1)$ & 0 & 0 & 0 & 0 & 0 & 0 & 0 & 0 & 0 & 0 & 0 \\
\hline,$- \quad 1$ & , $\quad 0$, & $-1)$ & 0 & 0 & 0 & 0 & 0 & 0 & 0 & 0 & 0 & 0 & 0 \\
\hline$(+,-2$, &,-2 , & 3) & 0 & 0 & 7 & 6 & -15 & 3 & 0 & 0 & 0 & 0 & 0 \\
\hline$(-,-1$, & , 3, & $-1)$ & 0 & 0 & -14 & -8 & -10 & -1 & 0 & 0 & 0 & 0 & 0 \\
\hline$(+,-4$ &,-1 , & & 0 & 0 & 0 & 0 & 0 & 0 & 0 & 1 & 0 & 0 & 0 \\
\hline$(-,-2$, &,-2 , & & 0 & 0 & 0 & 0 & 0 & 0 & 0 & 0 & 1 & 0 & 0 \\
\hline$(-, \quad 2$ &,-1 , & & -6 & 0 & -42 & -30 & 30 & 30 & -6 & -1 & 2 & 0 & 0 \\
\hline$(-,-2$, &,-1 , & 3) & 2 & 0 & 0 & 6 & -6 & -6 & -2 & -3 & -6 & 0 & 0 \\
\hline,$- \quad 2$, & , 0 , & $-1)$ & 6 & 0 & -28 & 20 & -20 & -20 & -12 & 6 & -6 & 0 & 0 \\
\hline$(+, 2$ & , 0 , & $-1)$ & -12 & 0 & -14 & -30 & 30 & 30 & 6 & 3 & -12 & 0 & 0 \\
\hline$(-,-2$, & , 2, & 2) & 0 & 0 & 0 & 0 & 0 & 0 & 0 & 0 & 0 & 1 & 0 \\
\hline$(+, \quad 2$ &,-1 , & $-1)$ & 0 & 0 & 0 & 0 & 0 & 0 & 0 & 0 & 0 & 0 & 2 \\
\hline$(-, \quad 2$ &,-1 , & $-2)$ & 6 & 0 & 0 & 0 & 0 & 0 & 0 & -4 & 0 & 4 & , \\
\hline$(-, \quad 0$ & 1 , & & 6 & -120 & 42 & 78 & 30 & -132 & -42 & -9 & -6 & -6 & 6 \\
\hline$(+, \quad 0$ & & & -48 & -125 & 0 & -81 & 0 & 0 & 0 & -3 & & -12 & 0 \\
\hline$(-,-1$, & , 1 , & 1) & 0 & 0 & 0 & 0 & 0 & -81 & 0 & 0 & 0 & 0 & 0 \\
\hline$(+, 1$ &,-1 & $-1)$ & 15 & -15 & -21 & 36 & 45 & 18 & -27 & -2 & -1 & -2 & \\
\hline$(-, \quad 1$ & 1, & 0) & 0 & 0 & -21 & -24 & 15 & 6 & 0 & 0 & 0 & 0 & \\
\hline
\end{tabular}




\subsubsection{Functional equations for $m=5$}

Table 2: Generators and constant $\lambda=\lambda\left(c_{j}^{(5)}\right)$ for $\operatorname{ker}\left(\beta_{5}^{\mathbf{Q}(t)}\right)^{\mathcal{S}_{3}}$ in Theorem 2

\begin{tabular}{|c|c|c|c|c|c|c|c|c|c|}
\hline$a$ & $c_{1}^{(5)}$ & $c_{2}^{(5)}$ & $c_{3}^{(5)}$ & $c_{4}^{(5)}$ & $c_{5}^{(5)}$ & $c_{6}^{(5)}$ & $c_{7}^{(5)}$ & $c_{8}^{(5)}$ & $c_{9}^{(5)}$ \\
\hline$(-, \quad 2,-2, \quad 3)$ & 18 & 0 & 0 & 0 & 0 & 0 & 0 & 0 & 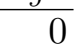 \\
\hline$(+, \quad 0, \quad 5, \quad 0)$ & 0 & 3 & 0 & 0 & 0 & 0 & 0 & 0 & 0 \\
\hline$(-, \quad 6,-1,-1)$ & 0 & 0 & 3 & 0 & 0 & 0 & 0 & 0 & 0 \\
\hline$(+, \quad 3, \quad 0, \quad 0)$ & 0 & 0 & 0 & 3 & 0 & 0 & 0 & 0 & 0 \\
\hline$(+, \quad 0,-3,3)$ & 0 & 0 & 0 & 0 & 10 & 0 & 0 & 0 & 0 \\
\hline$(-,-3, \quad 6,-3)$ & 0 & 0 & 0 & 0 & 0 & 3 & 0 & 0 & 0 \\
\hline$(-,-3, \quad 3,3)$ & 0 & 0 & -63 & -12 & 0 & 15 & 0 & 0 & 0 \\
\hline$(+, \quad 0,-5, \quad 5)$ & 0 & 0 & 0 & 0 & 0 & 0 & 3 & 0 & 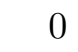 \\
\hline$(+, \quad 4,-1, \quad 0)$ & 0 & -18 & 0 & 0 & 0 & 0 & 6 & 0 & 0 \\
\hline$(+,-3, \quad 4, \quad 4)$ & 0 & 0 & 15 & 3 & 0 & -3 & 0 & 0 & 0 \\
\hline$(+, \quad 3, \quad 0,-2)$ & 0 & 12 & 0 & 0 & 0 & 0 & -54 & 0 & 0 \\
\hline$(-,-1, \quad 2,-1)$ & 0 & 0 & 0 & 0 & 0 & -243 & 0 & 0 & 0 \\
\hline$(+, \quad 0, \quad 1, \quad 1)$ & 0 & -144 & 0 & 0 & 0 & 0 & -27 & 0 & . \\
\hline$(-, \quad 2, \quad 0,-2)$ & 0 & 0 & 0 & 0 & 0 & 0 & 0 & 90 & 0 \\
\hline$(+, \quad 1, \quad 0,-1)$ & 0 & 0 & 0 & \multicolumn{2}{|c|}{$0-810$} & \multicolumn{2}{|c|}{$0-1875$} & 0 & 0 \\
\hline$(-, \quad 1, \quad 0,-1)$ & 0 & 0 & 0 & 0 & 0 & 0 & 0 & \multicolumn{2}{|c|}{0180} \\
\hline$(+,-2,-2, \quad 3)$ & 0 & 0 & 105 & 18 & 0 & -45 & 0 & 0 & 0 \\
\hline$(-,-1, \quad 3,-1)$ & 0 & 0 & -105 & -12 & 0 & -15 & 0 & 0 & 0 \\
\hline$(+,-4,-1, \quad 4)$ & -8 & -10 & -56 & -14 & 6 & 14 & 20 & -8 & 2 \\
\hline$(-,-2,-2, \quad 5)$ & 5 & 4 & 35 & 8 & 3 & -8 & 7 & -4 & 1 \\
\hline$(-, \quad 2,-1, \quad 1)$ & -80 & 80 & -1190 & -224 & 6 & 224 & -10 & -8 & 2 \\
\hline$(-,-2,-1, \quad 3)$ & 0 & 0 & 0 & 0 & -54 & 0 & -150 & \multicolumn{2}{|c|}{$72-18$} \\
\hline$(-, \quad 2, \quad 0,-1)$ & 180 & 360 & 1050 & 336 & -54 & -336 & -270 & \multicolumn{2}{|c|}{$72-18$} \\
\hline$(+, \quad 2, \quad 0,-1)$ & -360 & -180 & -2310 & -504 & -54 & 504 & -90 & \multicolumn{2}{|c|}{$72-18$} \\
\hline$(-,-2, \quad 2, \quad 2)$ & -40 & -20 & -112 & -28 & 12 & 28 & 40 & -1 & 4 \\
\hline$(+, \quad 2,-1,-1)$ & 0 & 0 & 0 & & -108 & -162 & -150 & -96 & -6 \\
\hline$(-, \quad 2,-1,-2)$ & 190 & 200 & 1120 & 280 & -120 & -280 & -400 & \multicolumn{2}{|c|}{$40-40$} \\
\hline$\left(\begin{array}{llll}-, & 0, & 1, & 0\end{array}\right)$ & 540 & -540 & 9450 & 1980 & -108 & -2142 & -720 & \multicolumn{2}{|c|}{$-36-36$} \\
\hline$(+, \quad 0, \quad 1, \quad 0)$ & -360 & -1425 & 2520 & 387 & -270 & -630 & -900 & \multicolumn{2}{|c|}{$0-90$} \\
\hline$(-,-1, \quad 1, \quad 1)$ & 0 & 0 & 5103 & 972 & & -1215 & 0 & 0 & 0 \\
\hline$(+, \quad 1,-1,-1)$ & -544 & -560 & 1085 & 32 & 39 & -194 & 370 & 8 & 13 \\
\hline$(-, \quad 1, \quad 1, \quad 0)$ & 0 & 0 & -315 & -72 & 0 & 45 & 0 & 0 & 0 \\
\hline & 0 & 0 & & 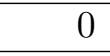 & 1662 & 378 & 4230 & 26 & 414 \\
\hline
\end{tabular}




\subsubsection{Functional equations for $m=6$ and $m=7$}

Table 3: Generators for $\operatorname{ker}\left(\beta_{7}^{\mathbf{Q}(t)}\right)^{\mathcal{S}_{3}}$ and $\operatorname{ker}\left(\beta_{6}^{\mathbf{Q}(t)}\right)^{\mathcal{S}_{3}}$ in Theorem 2

\begin{tabular}{|c|c|c|c|c|c|c|}
\hline$a$ & $c_{1}^{(6)}$ & $c_{2}^{(6)}$ & $c_{3}^{(6)}$ & $c_{4}^{(6)}$ & $c_{1}^{(7)}$ & $c_{2}^{(7)}$ \\
\hline$(-, \quad 2,-2, \quad 3)$ & 6 & 0 & 0 & 0 & 50 & 126 \\
\hline$(+, \quad 0, \quad 5, \quad 0)$ & 0 & 3 & 0 & 0 & -3 & 0 \\
\hline$(-, \quad 6,-1,-1)$ & 0 & 0 & 3 & 0 & 0 & 3 \\
\hline$(+, \quad 3, \quad 0, \quad 0)$ & 0 & 0 & 0 & 5 & 0 & -35 \\
\hline$(+, \quad 0,-3,3)$ & 0 & 0 & 0 & 0 & 0 & 140 \\
\hline$(-,-3, \quad 6,-3)$ & 0 & 0 & 21 & 4 & 0 & 7 \\
\hline$(-,-3, \quad 3, \quad 3)$ & 0 & 0 & -84 & -20 & 0 & 28 \\
\hline$(+, \quad 0,-5, \quad 5)$ & 0 & 0 & 0 & 0 & 4 & 0 \\
\hline$(+, 4,-1, \quad 0)$ & 0 & -30 & 0 & 0 & 50 & 0 \\
\hline$(+,-3, \quad 4, \quad 4)$ & 0 & 0 & 12 & 3 & 0 & -3 \\
\hline$(+, \quad 3, \quad 0,-2)$ & 0 & 60 & 0 & 0 & -300 & 0 \\
\hline$(-,-1, \quad 2,-1)$ & 0 & 0 & -5103 & -972 & 0 & -5103 \\
\hline$(+, \quad 0,1,1)$ & 0 & -360 & 0 & 0 & 900 & 0 \\
\hline$(-, \quad 2, \quad 0,-2)$ & 0 & 0 & 0 & 0 & -2750 & -4410 \\
\hline$(+, \quad 1, \quad 0,-1)$ & 0 & 0 & 0 & 0 & -62500 & -102060 \\
\hline$(-, \quad 1, \quad 0,-1)$ & 0 & 0 & 0 & 0 & -68000 & -112140 \\
\hline$(+,-2,-2, \quad 3)$ & 0 & 0 & -420 & -90 & 0 & -210 \\
\hline$(-,-1, \quad 3,-1)$ & 0 & 0 & -420 & -60 & 0 & -420 \\
\hline$(+,-4,-1, \quad 4)$ & 8 & 50 & 224 & 70 & 50 & 70 \\
\hline$(-,-2,-2, \quad 5)$ & 5 & 20 & 140 & 40 & 25 & 35 \\
\hline$(-, 2,-1, \quad 1)$ & -40 & 200 & -2380 & -560 & -1000 & -140 \\
\hline$(-,-2,-1, \quad 3)$ & -120 & -600 & -3402 & -1008 & -1000 & -1386 \\
\hline$(-, \quad 2, \quad 0,-1)$ & 180 & 1800 & 4200 & 1680 & -4500 & -7140 \\
\hline$(+, \quad 2, \quad 0,-1)$ & -360 & -900 & -9240 & -2520 & -4500 & -6720 \\
\hline$(-,-2, \quad 2, \quad 2)$ & -20 & -50 & -224 & -70 & -125 & -343 \\
\hline$(+, 2,-1,-1)$ & 20 & 600 & 0 & 360 & 3000 & -420 \\
\hline$(-, \quad 2,-1,-2)$ & -190 & -1000 & -4480 & -1400 & -250 & 490 \\
\hline$\left(\begin{array}{llll}-, & 0, & 1, & 0\end{array}\right)$ & 540 & -2700 & 37800 & 9900 & 27000 & -22680 \\
\hline$(+, \quad 0,1, \quad 0)$ & -360 & -7125 & 10080 & 1935 & 26625 & -22995 \\
\hline$(-,-1, \quad 1, \quad 1)$ & 0 & 0 & 20412 & 4860 & 0 & -20412 \\
\hline$(+, \quad 1,-1,-1)$ & 544 & 2800 & -4340 & -160 & 400 & -20272 \\
\hline$(-, 1,1,0)$ & 0 & 0 & -630 & -180 & 0 & 630 \\
\hline \multirow[t]{2}{*}{$\lambda$} & \multirow[t]{2}{*}{0} & \multirow[t]{2}{*}{0} & \multirow[t]{2}{*}{0} & \multirow[t]{2}{*}{0} & 25461 & 54495 \\
\hline & & & & & 4 & 4 \\
\hline
\end{tabular}




\subsection{Specializing to ladders}

A polylogarithmic ladder is a (finite) linear combination $\sum_{i} n_{i} L i_{m}\left(\alpha^{i}\right)$ for some algebraic number $\alpha$, some positive integer $m$, and integers $n_{i}$, which can be written as a rational linear combination of products of logarithms. Lewin gave numerical examples up to weight $m=9$ (cf. [1], Chapters 1-6 in [25]). Cohen, Lewin and Zagier were able to push the set-up in Zagier's polylogarithm conjecture [31] to produce a numerical example of a ladder up to weight $m=16$ (cf. [9]), but they had missed a cyclotomic relation (of degree 630) that was eventually detected by Bailey and Broadhurst, allowing the latter to "climb" one weight higher to the current ladder record $m=17$ (cf. [2], where they also give ladders for other Salem numbers up to weight 13). The algebraic number $\alpha$ involved in this ladder is a very distinguished one: it is the so-called Lehmer number (the unique root of $x^{10}+x^{9}-x^{7}-$ $x^{6}-x^{5}-x^{4}-x^{3}+x+1$ of absolute value $>1$ ) which conjecturally has the smallest Mahler measure among algebraic numbers.

The originally quite surprising occurrence of such ladders seems to be well understood now in the context of Zagier's polylogarithm conjecture (see, e.g., [31], Section 7C and [32], Section 4).

2.5.1. New ladders of weight 6 and 7. From the functional equations above, we can deduce four linearly independent ladders of weight 6 and two of weight 7 . We give the latter ones here.

With the notation of [1], we let $\omega$ be a root of the equation

$$
x^{3}+x^{2}=1 .
$$

Zagier's conjecture implies that there should be at least 4 linearly independent ladders for weight 7 for $\omega$ (cf. [9], Section 3, and [32], Section 4).

By substituting $-\omega$ for $t$ in the two independent functional equations in one variable stated in table 3 in terms of the coefficients $c_{j}^{(7)}=\left\{c_{j}^{(7)}(a)\right\}$ $(j=1,2)$, we arrive at the first proven ladder relations for weight 7 . We have divided the coefficients by a suitable power of 2 for ease of reading.

Corollary 4. Let $\alpha$ and $\beta$ denote the following two ladders

$$
\begin{aligned}
\alpha= & \frac{35397}{256}[1]+\frac{1475}{8}[\omega]-\frac{166525}{1024}\left[\omega^{2}\right]-\frac{3825}{16}\left[\omega^{3}\right]-\frac{55025}{512}\left[\omega^{4}\right]+127\left[\omega^{5}\right] \\
& +\frac{34575}{512}\left[\omega^{6}\right]-\frac{5225}{256}\left[\omega^{8}\right]+\frac{475}{16}\left[\omega^{9}\right]-\frac{4117}{1024}\left[\omega^{10}\right]-\frac{1375}{512}\left[\omega^{12}\right]-\frac{75}{8}\left[\omega^{14}\right] \\
& -\frac{29}{16}\left[\omega^{15}\right]-\frac{475}{1024}\left[\omega^{18}\right]-\frac{133}{512}\left[\omega^{20}\right]+\frac{25}{256}\left[\omega^{28}\right]+\frac{29}{1024}\left[\omega^{30}\right]
\end{aligned}
$$


and

$$
\begin{aligned}
\beta= & \frac{194355}{512}[1]+\frac{6265}{16}[\omega]-\frac{479395}{1024}\left[\omega^{2}\right]-\frac{2317}{4}\left[\omega^{3}\right]-\frac{146125}{1024}\left[\omega^{4}\right]+\frac{5005}{16}\left[\omega^{5}\right] \\
& +\frac{84455}{512}\left[\omega^{6}\right]-9\left[\omega^{7}\right]-\frac{6769}{128}\left[\omega^{8}\right]+\frac{497}{16}\left[\omega^{9}\right]-\frac{9835}{1024}\left[\omega^{10}\right]-\frac{5523}{1024}\left[\omega^{12}\right] \\
& -\frac{1551}{128}\left[\omega^{14}\right]-\frac{35}{16}\left[\omega^{15}\right]-\frac{497}{1024}\left[\omega^{18}\right]-\frac{245}{1024}\left[\omega^{20}\right]+\frac{65}{512}\left[\omega^{28}\right]+\frac{35}{1024}\left[\omega^{30}\right] .
\end{aligned}
$$

Then

$$
\mathcal{L}_{7}(\alpha)=\mathcal{L}_{7}(\beta)=0
$$

We note that from the 2-variable equation for the 7-logarithm in [16] we do not obtain an independent ladder, but instead a linear combination of these two, viz.

$$
\begin{aligned}
& \mathcal{L}_{7}\left(-\frac{476217}{512}[1]-\frac{10675}{16}[\omega]+\frac{307825}{256}\left[\omega^{2}\right]+\frac{19565}{16}\left[\omega^{3}\right]-\frac{39725}{1024}\left[\omega^{4}\right]-\frac{10801}{16}\left[\omega^{5}\right]\right. \\
& \quad-\frac{90125}{256}\left[\omega^{6}\right]+45\left[\omega^{7}\right]+\frac{31115}{256}\left[\omega^{8}\right]+\frac{105}{2}\left[\omega^{9}\right]+\frac{5089}{256}\left[\omega^{10}\right]+\frac{8365}{1024}\left[\omega^{12}\right] \\
& \left.\quad-\frac{645}{128}\left[\omega^{14}\right]-\frac{7}{4}\left[\omega^{15}\right]-\frac{105}{128}\left[\omega^{18}\right]-\frac{637}{1024}\left[\omega^{20}\right]+\frac{25}{512}\left[\omega^{28}\right]+\frac{7}{256}\left[\omega^{30}\right]\right)=0 .
\end{aligned}
$$

This seems to suggest that the 2 -variable equation just mentioned may not specialize (at least not directly) to the individual 1-variable equations for $\mathcal{L}_{7}$ in table 3 .

We can corroborate here a certain "correlation" of exponents and coefficients which had already been observed by Lewin in connection with other ladders: denoting by $\alpha_{k}$ and $\beta_{k}$ the coefficient of $\left[\omega^{k}\right]$ in the ladders $\alpha$ and $\beta$ given above, we find for $k>0$ that

$$
5\left|\alpha_{k} \Leftrightarrow 5 \not k, \quad 7\right| \beta_{k} \Leftrightarrow 7 \not k .
$$

\section{Acknowledgments}

We are grateful to Don Zagier for invaluable advice and to David Broadhurst and an unanonymous referee for useful comments.

\section{References}

[1] M. Abouzahra, L. Lewin and Hongnian Xiao, Polylogarithms in the field of omega (a root of a given cubic): functional equations and ladders, Aequationes Math. 33(1) (1987), 1-20. 
[2] D. Bailey and D. Broadhurst, A seventeenth-order polylogarithm ladder, preprint 1999, http://arxiv.org/abs/math.CA/9906134.

[3] S. Bloch, Higher regulators, algebraic K-theory, and zeta functions of elliptic curves, CRM Monograph Series 11, American Mathematical Society, Providence, RI, 2000 (known also as "Irvine Lecture Notes", 1978).

[4] D. Broadhurst, A dilogarithmic 3-dimensional Ising tetrahedron, The Eur. Phys. J. C, 8(2) (1999), 363-366.

[5] D. Broadhurst, Massive 3-loop Feynman diagrams reducible to $S C^{*}$ primitives of algebras of the sixth root of unity, Eur. Phys. J. C 8 (1999), 311-333.

[6] D. Broadhurst and D. Kreimer, Association of multiple zeta values with positive knots via Feynman diagrams up to 9 loops, Phys. Lett. B 393 (1997), 403-412.

[7] F. Brown, The massless higher-loop two point function, Commun. Math. Phys. 287(3) (2009), 925-958; arXiv:0804.1660.

[8] S. Caron-Huot, Superconformal symmetry and two-loop amplitudes in planar N=4 super Yang-Mills, J. High Energy Phys. 12 (2011), 066; arXiv:1105.5606 [hep-th].

[9] H. Cohen, L. Lewin and D. Zagier, A sixteenth-order polylogarithm ladder, Exp. Math. 1 (1992), 25-34.

[10] V. Del Duca, C. Duhr and V.A. Smirnov, The Two-Loop Hexagon Wilson Loop in $\mathcal{N}=4 S Y M$; arXiv:1003.1702 [hep-th].

[11] V. Del Duca, L.J. Dixon, J.M. Drummond, C. Duhr, J.M. Henn and V.A. Smirnov, The one-loop six-dimensional hexagon integral with three massive corners, Phys. Rev. D 84 (2011), 045017; arXiv:1105.2011 [hep-th].

[12] L.J. Dixon, J.M. Drummond and J.M. Henn, Bootstrapping the threeloop hexagon, J. High Energy Phys. 11, 023 (2011); arXiv:1108.4461 [hep-th].

[13] L.J. Dixon, C. Duhr and J. Pennington, Single-valued harmonic polylogarithms and the multi-Regge limit, J. High Energy Phys. 10 (2012), 074; arXiv: 1207.0186 [hep-th]. 
[14] H. Gangl, Funktionalgleichungen von Polylogarithmen, Bonner Math. Schriften 278 (1995), Thesis Universität Bonn.

[15] H. Gangl, Families of functional equations for polylogarithms, Algebraic K-theory (Poznan, 1995), Contemp. Math., 199, Amer. Math. Soc., Providence, RI, 1996, 83-105.

[16] H. Gangl, Functional equations for higher logarithms, Selecta Math. (N.S.) $9(3)$ (2003), 361-377.

[17] J. Golden, A.B. Goncharov, M. Spradlin, C. Vergu and A. Volovich, Motivic Amplitudes and Cluster Coordinates; arXiv:1305.1617 [hep-th].

[18] A.B. Goncharov, Geometry of configurations, polylogarithms and motivic cohomology, Adv. Math. 114 (1995), 179-319.

[19] A.B. Goncharov, Galois symmetries of fundamental groupoids and noncommutative geometry, Duke Math. J. 128(2) (2005), 209-284; arXiv:math/0208144.

[20] A.B. Goncharov, M. Spradlin, C. Vergu and A. Volovich, Classical Polylogarithms for Amplitudes and Wilson Loops, Phys. Rev. Lett. 105 (2010), 151605; arXiv:1006.5703 [hep-th].

[21] G. t'Hooft and M. Veltman, Regularization and renormalization of gauge fields, Nucl. Phys. B 44 (1972), 189-213.

[22] M.Yu. Kalmykov, Gauss hypergeometric function: reduction, E-expansion for integer/half-integer parameters and Feynman diagrams, J. High Energy Phys. 4 (2006), 056.

[23] E. Kummer, Über die Transzendenten, welche aus wiederholten Integralen rationaler Funktionen entstehen, J. Reine Angew. Math. 21 (1840), 74-90, 193-225, 328-371.

[24] L. Lewin, Polylogarithms and associated functions, North-Holland, New York, 1981.

[25] L. Lewin, Structural properties of polylogarithms, Mathematical Surveys and Monographs 37, AMS, Providence, RI.

[26] L. Lewin and E. Rost, Polylogarithmic functional equations: A new category of results developed with the help of computer algebra (MACSYMA), Aequationes Math. 31 (1986), 212-221. 
[27] W. Nahm, Conformal Field Theory and Torsion Elements of the Bloch Group, in "Frontiers in Number Theory, Physics and Geometry II", Les Houches Proceedings, Springer (Cartier, Julia, Moussa, Vanhove, eds.) (2007), 67-132.

[28] V.A. Smirnov, Analytical Result for Dimensionally Regularized Massive On-Shell Planar Double Box, Phys. Lett. B 524 (2002), 129-136.

[29] N.I. Ussyukina and A.I. Davydychev, Exact results for three- and fourpoint ladder diagrams with an arbitrary number of rungs, Phys. Lett. B 305(1-2) (1993), 136-143.

[30] G. Wechsung, Über die Unmöglichkeit des Vorkommens von Funktionalgleichungen gewisser Struktur für Polylogarithmen, Aequationes Math. 5 (1970), 54-62.

[31] D. Zagier, Polylogarithms, Dedekind zeta functions and the algebraic K-theory of fields, Arithmetic algebraic geometry (Texel, 1989), Progr. Math. 89, Birkhäuser (1991), 391-430.

[32] D. Zagier, Special values and functional equations of polylogarithms, Appendix A to "Structural properties of polylogarithms", [25].

Department of Mathematical Sciences

DURHAM UNIVERSITY

SOUTH ROAD

DURHAM DH1 3LE

UK

E-mail address: Herbert.Gangl@durham.ac.uk

Received September 17, 2010 